\title{
The net reproduction rate and the type- reproduction number in multiregional demography
}

\author{
Hisashi Inaba*
}

\begin{abstract}
In order to study effects of migration on demographic changes of multiregional populations, multiregional population modelling is a useful traditional tool. Although multiregional mathematical demography has been extensively explored since the beginning of the 1970s, its key concept, the multiregional net reproduction rate, has been long neglected. In this review, we focus on a multiregional stable population system and elaborate the definition of the multiregional net reproduction rate. Next we introduce the type-reproduction number from mathematical epidemiology and show that it becomes a useful index to formulate a simple control relation for a multiregional population. Mathematical ideas presented here will help us to reconsider multiregional mathematical demography, which is a useful theoretical framework to study effects of interregional migration on population dynamics and composition.
\end{abstract}

\section{Introduction}

Multiregional population modelling is a traditional tool used to study effects of migration on population dynamics and composition. In fact, the multiregional (more generally speaking, multistate) approach has been used in the literature in the context of international migration and its long-run effects on multinational population system (Coleman 2006). From a theoretical point of view, multiregional life table, multiregional stable population model and multiregional Leslie matrix are basic tools to allow for a consistent multiregional (multistate) population projection under the Markovian assumption for interregional migration. Those techniques were extensively developed and became very popular through the IIASA project "Migration and Settlement" (1976-1983) under the leadership of Andrei Rogers. In many cases, the multiregional population

* Hisashi Inaba, Graduate School of Mathematical Sciences, University of Tokyo, 3-8-1 Komaba Meguro-ku Tokyo, Japan 153-8914. Email: inaba@ms.u-tokyo.ac.jp

DOI: 10.1553/populationyearbook2009s197 
projection model focused on the quantitative assessment of internal migration patterns and spatial population dynamics in each country, and it is clear that as long as a bottleneck of data availability is dissolved the multiregional demographic method can be applied to study effects of international migration on multinational population systems as was shown early on by Rogers and Willekens (1978). Looking at the age of the IIASA project, computing power has since made rapid progress, Eurostat is gradually developing its dataset and apparently aims at including migration flows within Europe, so improving the availability of data on international migration flows will eventually make it possible to use multiregional demographic modelling for the study of international migration. Some important aspects of the multistate demographic theory, however, are still not yet properly developed. This applies in particular to the concept of net reproduction rate, which proved to be a key idea in the classical (single-state) stable population model; the concept has not been properly extended to the multiregional case yet, although its definition was already suggested by Rogers and Willekens in 1978 (Willekens 1977; Rogers and Willekens 1978). In this paper, our purpose is to address this deficiency and to suggest the potential of multiregional population dynamics models by which we examine effects of migration and consider population policy for multinational population systems.

First we briefly sketch the historical development of the idea of the multistate net reproduction rate. Next we elaborate the general definition of a multiregional net reproduction rate based on the multiregional stable population model. Finally we introduce a new index, called the type-reproduction number, developed in mathematical epidemiology, and discuss its typical demographic application for the calculation of the critical fertility level to maintain a stationary multinational population. Although in this note we do not discuss substantive migration phenomena, the mathematical tools presented here will help us to reconsider multiregional mathematical demography, which is a useful theoretical framework to study effects of interregional migration on whole population dynamics and composition.

\section{Historical review of the net reproduction rate}

The net reproduction rate, denoted by $R_{0}$, is one of most important indices in mathematical demography. The quantity is defined as the expected number of female newborns produced by a woman during her entire life. Under the regime of classical (single-state or uni-regional) stable population theory, $R_{0}$ is calculated as

$$
R_{0}=\int_{0}^{\infty} \beta(a) \ell(a) d a,
$$


where $\beta(a)$ is the maternity function (the age-specific rate of having a female birth), $\ell(a)$ is the female's survival rate at age $a$. Then $\psi(a):=\beta(a) \ell(a)$ is called the net maternity function and its normalised distribution $\psi_{0}(a):=\psi(a) / R_{0}$ gives the probability density of age at childbearing.

Another crucial index is the asymptotic growth rate, denoted by $\lambda_{0}$, of the number of newborns of a closed population with time-independent vital rates, called the intrinsic rate of natural increase. According to the classical stable population theory (Dublin and Lotka 1925; Lotka 1998; Keyfitz and Caswell 2005), $\lambda_{0}$ is a unique real root of the Lotka's characteristic equation:

$$
\int_{0}^{\infty} e^{-\lambda a} \psi(a) d a=1
$$

Then it is proved that $\lambda_{0}>0$ if $R_{0}>1, \lambda_{0}=0$ if $R_{0}=1$ and $\lambda_{0}<0$ if $R_{0}<1$. Therefore the net reproduction rate $R_{0}$ formulates the threshold condition for population growth based on parameters capturing the average behaviour of individuals, i.e. it connects individual life cycle parameters to the growth character of the whole population.

On the other hand, if once we try to take into account many facets of heterogeneity at individual level, the classical stable population theory should be extended to the multistate stable population theory. ${ }^{\text {I }}$ In fact, a real-world population is divided into many regions of residence and connected by interregional migration, and each regional population has its own vital rates. In such a case, the classical stable population model relying on a homogeneous population or an averaged person is insufficient to reflect the diversity of individuals and their heterogeneous behaviour. In his early book in 1975 as well as in its update from 1995 (Rogers 1975; Rogers 1995), Andrei Rogers developed a multiregional extension of the stable population model, ${ }^{2}$ and defined the net reproduction matrix ${ }^{3}$ as follows (Rogers 1975, p.106):

$$
K:=\int_{0}^{\infty} \Psi(a) d a=\int_{0}^{\infty} M(a) L(a) d a
$$

1 Although here we treat a case in which the state space of the parameter describing individual heterogeneity is finite-dimensional, we can also construct a stable population theory with infinite dimensional parameter space (Metz and Diekmann 1986). Moreover, as we focus on a closed multistate population system here, it is possible to extend the model to take into account immigration from exogeneous source (Inaba 1988b).

2 The idea of multiregional stable population originated in his earlier paper and book published in 1966 and 1968 (Rogers 1966; Rogers 1968). As far as I know, Le Bras (1971) independently formulated the continuous-time multiregional stable population model. The reader may also refer to Land and Rogers (1982) for historical aspects of multistate demography.

3 Rogers (1995) called $K$ the net reproduction rate matrix, but we are consistently using the term 'net reproduction matrix'. 
where $M(a)$ is an $N$-dimensional diagonal matrix whose $(i, i)$-th entry $m_{i}(a)$ denotes the maternity function in $i$-th region, ${ }^{4} L(a)=\left(\ell_{i j}(a)\right)_{1 \leq i, j \leq N}$ is the survival rate matrix whose $(i, j)$-th entry $\ell_{i j}(a)$ denotes the probability that a baby girl born in region $j$ will survive to age $a$ in region $i$ and $\Psi(a):=M(a) L(a)$ is called the multiregional net maternity function. Let $k_{i j}$ be the $(i, j)$-th entry of $K$. Then $k_{i j}$ is the expected number of female newborns in region $i$ produced by a woman born in region $j$ during her entire life.

The Laplace transform of the net maternity function

$$
\widehat{\Psi}(\lambda):=\int_{0}^{\infty} e^{-\lambda a} \Psi(a) d a,
$$

is defined as the multiregional characteristic matrix. Though his mathematical argument was not necessarily rigorous, ${ }^{5}$ Rogers stated that the intrinsic rate of natural increase of a multiregional stable population is given by the real number $\lambda_{0}$ such that

$$
r\left(\widehat{\Psi}\left(\lambda_{0}\right)\right)=1
$$

where $r(A)$ denotes the spectral radius ${ }^{6}$ of a matrix $A$. From the well-known Perron-Frobenius theory, $r(A)$ becomes a nonnegative eigenvalue when $A$ is a nonnegative matrix, and it is the largest positive eigenvalue of $A$ associated with a positive eigenvector when $A$ is irreducible. ${ }^{7}$ That is, Equation (2.2) implies that there exists a positive vector $u_{\lambda_{0}}$ such that

$$
\left(I-\int_{0}^{\infty} e^{-\lambda_{0} a} \Psi(a) d a\right) u_{\lambda_{0}}=0
$$

where $I$ is $N \times N$ identity matrix when $\widehat{\Psi}\left(\lambda_{0}\right), \lambda_{0} \in \mathbb{R}$ is irreducible. Equation (2.3) is a multiregional analogue of the characteristic Equation (2.1).

Rogers and Willekens, however, did not necessarily consistently define the multiregional version of the net reproduction rate $R_{0}$ by which the threshold

$4 \quad$ The diagonal fertility matrix implies that newborns and their mothers belong to the same state. This assumption is reasonable for multiregional models, but it is not necessarily the case for other multistate models.

5 The reader may consult Inaba $(1987,1988$ a) for a detailed proof.

$6 r(A)=\max _{\lambda \in \sigma(A)}|\lambda|$ where $\sigma(A)$ denotes the set of eigenvalues of $A$ and it follows that $r(\mathrm{~A})=\lim _{n \rightarrow \infty}\left\|A^{n}\right\|^{1 / n}$.

$7 \quad$ A $N \times N$ matrix $A=\left(a_{i j}\right)_{1 \leq i, j \leq N}$ is called reducible (decomposable) if the set of numbers $L=\{1$, $2, \ldots, N\}$ is the sum of two disjoint subsets $K$ and $H$ such that $L=K \cup H, a_{i j}=0$ if $i \in K$ and $j \in$ $H$. If a matrix is not reducible, it is called irreducible (indecomposable). If $A$ is irreducible and let $A^{n}=\left(a_{i j}^{(n)}\right)_{1 \leq i, j \leq N}$, for any $i$ and $j$ there exists an integer $n_{0}$ such that $a_{i j}^{\left(n_{0}\right)}>0$, so any one state is accessible from any other state by multiple transitions (Minc 1988). 
condition for population growth should be formulated. In a book published in 1986 (Rogers and Willekens 1986, Chapter 9, p. 378), Rogers and Willekens called $k_{i j}$ the 'spatial net reproduction rate', but it is a misleading definition because each region-dependent reproduction number $k_{i j}$ can tell us nothing about the threshold of population growth. On the other hand, Rogers and Willekens (1978) argued that for a spatial zero-growth population to be maintained, the dominant eigenvalue of the net reproduction matrix (which is given by $r(K)$ ) must be unity. Moreover, they argued,

A multiregional population system that is growing at a positive rate of growth exhibits a net-reproduction matrix $\mathbf{R}(0)$ with a dominant characteristic root $\lambda_{1}[\mathbf{R}(0)]$ that is greater than unity. [Rogers and Willekens (1978), p. 505]

This observation suggests that the multiregional net reproduction rate may be given by the spectral radius (the dominant positive eigenvalue) of the net reproduction matrix. Afterwards Rogers (1995, pp. 128-129) again stated that 'an overall net reproduction rate' is the dominant characteristic root of the net reproduction matrix, but did not elaborate his idea of 'overall net reproduction rate' any further.

On one hand, Inaba $(1987,1988 \mathrm{a})$ proved that $\lambda_{0}>0$ if $r(K)>1, \lambda_{0}=0$ if $r(K)$ $=1$ and $\lambda_{0}<0$ if $r(K)<1$ for the continuous-time multistate stable population model, ${ }^{8}$ and stated that the multiregional analogue of the net reproduction rate is $r(K)$. However, this result also had no influence on the later course of multistate demography, which mainly focused on practical methods for multiregional population projection and comparative quantitative assessment of migration patterns and spatial population dynamics; up to now, the concept of multistate net reproduction rate has been given less attention by demographers. ${ }^{9}$

It is regrettable that the multistate $R_{0}$ is not being used in multiregional demographic analysis. In fact, although many EU countries and USA now adopt the multiregional demographic model for their population projection purposes, if we do not use the multiregional $R_{0}$, we cannot discuss the intrinsic reproductive potential of the multiregional population, and it is also difficult to argue population policy quantitatively, taking into account regional heterogeneity.

In the field of mathematical epidemiology, Diekmann et al. (1990) established a general definition of the basic reproduction number $R_{0}$ for the spread of infectious diseases. The basic reproduction number in epidemiology originates from the concept of the net reproduction rate in demography (Dietz 1993;

8 The corresponding threshold result and the definition of $R_{0}$ for the generalised Leslie matrix model are given in Inaba (1986).

9 In fact, typical (mathematical) demographic texts such as Schoen (1988), Preston et al. (2001) and Keyfitz and Caswell (2005) do not refer to the multistate net reproduction rate although they introduce multistate demography. On the other hand, the reader may refer to Inaba (1995) and Billari et al. (2000) for realistic examples where $R_{0}$ is extended to multistate demographic models. 
Heesterbeek 2002), and it is defined as the expected number of secondary cases produced by a typical infected individual in a totally susceptible host population during its entire infectious period. If the number of states of an individual at 'birth' (in the epidemiological sense, i.e. at a new infection) is finite, the basic reproduction number is given by the dominant positive eigenvalue (the spectral radius) of the next generation matrix $K=\left(k_{i j}\right)_{1 \leq i, j \leq N}$ whose $(i, j)$-th entry $k_{i j}$ denotes the expected number of $i$-th secondary cases produced by a new case in state $j$ in a totally susceptible host population during its entire infectious period. Those developments in mathematical epidemiology clearly suggest that the multistate net reproduction rate in demography also should be the positive dominant eigenvalue (the spectral radius) of the net reproduction matrix $K$.

As suggested by the recent rapid developments in mathematical epidemiology after introducing the appropriate definition of $R_{0}$, neglecting the (multistate) net reproduction rate is a serious obstacle for theoretical developments in multistate demography, because the multistate net reproduction rate is the key concept which combines life cycle parameters capturing individual heterogeneity with macro population dynamics, hence it is essential to formulate population control criteria based on the heterogeneity of individual behaviour.

\section{The net reproduction rate for a multiregional stable population}

In this section, we elaborate on the idea of multiregional net reproduction rate. ${ }^{10}$ In the following, we mainly deal with 'multiregional' populations instead of 'multistate' populations in order to focus on the application to a multiregional system with inter-regional migration, although the reader may easily understand that the basic idea is not restricted to multiregional systems, which are just one example of more general multistate population models. ${ }^{11}$

Suppose that a large-scale closed female population were divided into $N$ regions. Let $p_{j}(t, a)(1 \leq j \leq N)$ be the age density of $j$-th regional population at time $t$ and age $a$. Let $p(t, a):=\left(p_{1}(t, a), \ldots, p_{N}(t, a)\right)^{\mathrm{T} 12}$ be an $N$-dimensional column vector of the multiregional population at time $t$. By using the multiregional survival rate matrix $L(a)$, the population vector $p(t, a), t \geq 0$ is expressed as follows:

10 Although the net reproduction rate is in fact not a 'rate' but a non-dimensional number or ratio, in this paper we use the conventional term 'rate'.

11 In general, we remark that the state-dependency often requires assumptions different from that of the regional dependency.

12 T denotes the transpose of the vector. 


$$
p(t, a)= \begin{cases}L(a) B(t-a), & t-a>0 \\ L(a) L^{-1}(a-t) p_{0}(a-t), & a-t>0\end{cases}
$$

where $B(t):=p(t, 0)$ is the number (vector) of newborns per unit time at time $t$ and $p_{0}(a):=p(0, a)$ is the initial data. Let $M(a)$ be an $N \times N$ diagonal fertility rate matrix, whose $i$-th diagonal element is the age-specific fertility rate of $i$-th region.

Then we have

$$
B(t)=\int_{0}^{\infty} M(a) p(t, a) d a .
$$

Inserting the Expression (3.1) into the boundary condition (3.2), we obtain the multiregional renewal equation (Lotka's integral equation) as ${ }^{13}$

$$
B(t)=G(t)+\int_{0}^{t} \Psi(a) B(t-a) d a, \quad t>0,
$$

where $\Psi(a)=M(a) L(a)$ and $G$ is given by

$$
G(t)=\int_{t}^{\infty} \Psi(a) L^{-1}(a-t) p_{0}(a-t) d a .
$$

If we define the $m$-th generation of newborns, denoted by $B_{m}(t)$, iteratively as

$$
B_{1}(t)=G(t),
$$

$$
B_{m+1}(t)=\int_{0}^{t} \Psi(a) B_{m}(t-a) d a, \quad m=1,2, \ldots,
$$

then $B_{1}(t)$ denotes the number of daughters produced by the initial population per unit time at time $t, B_{2}(t)$ is the number of granddaughters (of the initial population) per unit time at time $t$ and so on. It is well known that the solution of the renewal Equation (3.3) is given by

$$
B(t)=\sum_{m=1}^{\infty} B_{m}(t) .
$$

Integrating the iterative relation (3.4) and changing the order of integrals, we observe that

13 In the following we assume $\Psi$ to be a nonnegative integrable (matrix valued) function on $[0$, $\infty$ ), which is a reasonable assumption for our purpose because $M(a)$ is zero outside of finite reproductive period. 


$$
\int_{0}^{\infty} B_{m+1}(t) d t=\int_{0}^{\infty} \int_{0}^{t} \Psi(a) B_{m}(t-a) d a d t
$$

$$
\begin{aligned}
& =\int_{0}^{\infty} d a \int_{a}^{\infty} \Psi(a) B_{m}(t-a) d t \\
& =\int_{0}^{\infty} \Psi(a) d a \int_{0}^{\infty} B_{m}(t) d t .
\end{aligned}
$$

If we define

$$
X_{m}:=\int_{0}^{\infty} B_{m}(t) d t, \quad m \geq 1,
$$

then $X_{m}$ denotes the size distribution of $m$-th generation (the total number of offspring belong to $m$-th generation). Therefore it follows from (3.5) that

$$
X_{m+1}=K X_{m}=K^{m} X_{1}, \quad m=1,2, \ldots,
$$

where

$$
K=\int_{0}^{\infty} \Psi(a) d a
$$

is the multiregional net reproduction matrix. ${ }^{14}$

If the multiregional net reproduction matrix $K$ is a primitive matrix, ${ }^{15}$ its spectral radius $r(K)$ becomes the dominant positive eigenvalue of $K$, i.e. $|\lambda|<r(K)$ for any eigenvalue $\lambda$ of $K$ other than $r(K)$. Moreover it can be proved that

$$
\lim _{m \rightarrow \infty} r(K)^{-m} X_{m}=\frac{v_{0}^{T} X_{1}}{v_{0}^{T} u_{0}} u_{0},
$$

where $u_{0}$ and $v_{0}$ are the right and left positive eigenvectors of $K$ associated with the dominant eigenvalue $r(K)$.

As (3.6) shows that the size of each generation is asymptotically expanding or shrinking with the geometric growth rate $r(K)$, hence it is reasonable that we define the multiregional net reproduction rate $R_{0}$ as

$$
R_{0}=r(K) \text {. }
$$

Let $k_{i j}$ be the $(i, j)$-th entry of the net reproduction matrix $K$. Then

14 In mathematical epidemiology, $K$ is called the next generation matrix and $R_{0}=r(K)$ is called the basic reproduction number (Diekmann and Heesterbeek 2000), because $K$ maps a distribution (vector) of primary case to a distribution of secondary case.

15 A nonnegative matrix is primitive if and only if there exists an integer $n$ such that $A^{n}$ is strictly positive. 


$$
R_{j}=\sum_{i=1}^{N} k_{i j}
$$

is the total number of female offspring of a woman born in region $j$, so we may call it the native-dependent net reproduction rate, which is a cohort net reproduction rate of a woman born in region $j$. As was pointed out by Rogers and Willekens (1978), $R_{0}=r(K)=1$ if $R_{j}=1$ for all $j$, which is a most simple criterion for attaining the multiregional population replacement level. It is also well known in mathematical economics that $R_{0}=r(K)<1$ if $\max _{j} R_{j}<1$ (Brauer-Solow condition), or if $I-K$ has $N$ positive upper left-hand corner principal minors (Hawkins-Simon condition). Although according to neglect of the multiregional $R_{0}$, there are not so many examples of the net reproduction matrix $K$ estimated from real data, Rogers and Willekens (1978) gave the two-regional net reproduction matrix for urban-rural data for India, the Soviet Union and the USA. The urban-rural net reproduction matrix for India in 1970 is computed as

$$
K=\left(\begin{array}{ll}
1.10 & 0.25 \\
0.50 & 1.55
\end{array}\right) \text {, }
$$

where Region one denotes urban area and Region two denotes rural area. Then the native-dependent net reproduction rate of urban area is $R_{1}=k_{11}+k_{21}=1.6$, while for rural area $R_{2}=k_{12}+k_{22}=1.8$. The two-regional net reproduction rate $R_{0}$ $=r(K)$ is 1.74 .

As we see above, the net reproduction rate formulates the threshold condition for population growth in the generational perspective, but we still have to check whether it also gives the threshold condition for population growth in real time. In fact, we can show that the intrinsic rate of natural increase is given by the real number $\lambda_{0}$ satisfying (2.2) and it follows that

$$
\lim _{t \rightarrow \infty} e^{-\lambda_{0} t} B(t)=Q_{0} u_{\lambda_{0}},
$$

where the coefficient $Q_{0}$ is given by

$$
Q_{0}=\frac{v_{\lambda_{0}}^{T} \hat{G}\left(\lambda_{0}\right)}{v_{\lambda_{0}}^{T} \widehat{\Psi}_{1}\left(\lambda_{0}\right) u_{\lambda_{0}}}, \quad \hat{G}\left(\lambda_{0}\right)=\int_{0}^{\infty} e^{-\lambda_{0} t} G(t) d t,
$$

$u_{\lambda_{0}}$ and $v_{\lambda_{0}}$ are the right and the left positive eigenvectors of $\widehat{\Psi}\left(\lambda_{0}\right)$ associated with eigenvalue unity and

$$
\widehat{\Psi}_{1}\left(\lambda_{0}\right)=-\left.\frac{d}{d \lambda} \widehat{\Psi}(\lambda)\right|_{\lambda=\lambda_{0}}=\int_{0}^{\infty} a e^{-\lambda_{0} a} \Psi(a) d a .
$$

Since $r(\widehat{\Psi}(\lambda))$ is strictly decreasing with respect to $\lambda \in \mathbb{R}$ and $R_{0}=r(\widehat{\Psi}((0))=$ $r(K)$, we obtain the threshold result that $\lambda_{0}>0$ if $R_{0}>1, \lambda_{0}=0$ if $R_{0}=1$ and $\lambda_{0}<0$ if $R_{0}<1$.

Define the multiregional stable age distribution by 


$$
\phi_{\lambda_{0}}(a):=e^{-\lambda_{0} a} L(a) u_{\lambda_{0}} .
$$

Then it follows from (3.1) and (3.7), we can prove that the following holds uniformly for any finite age interval:

$$
\lim _{t \rightarrow \infty} e^{-\lambda_{0} t} p(t, a)=Q_{0} \phi_{\lambda_{0}}(a) .
$$

Then the multiregional age profile converges to a multiregional stable age distribution as time evolves. This is the strong ergodicity theorem for the multiregional stable population model. ${ }^{16}$

\section{The type-reproduction number and control criteria}

As is discussed above, the idea of the multiregional (multistate) net reproduction rate has been long neglected and underutilised by demographers. However, without the net reproduction rate we cannot formulate the control measure for population growth in a heterogeneous population. In contrast to demography, mathematical epidemiology has successfully developed mathematical models dealing with heterogeneous population for disease prevention policy since the introduction of the general definition of the basic reproduction number. Here we consider applications of epidemic ideas based on the basic reproduction number and the type-reproduction number to demographic problems.

Using the net reproduction matrix $K$, we can consider effects of fertility changes on the net reproduction rate. The most basic question is how to control the entries of $K$ so that the multiregional net reproduction rate becomes unity, i.e. how we can lead to a stationary population, because it is a sustainable state in the long run. If we consider a homogeneous (uni-regional) population with $R_{0}>1$ and $\epsilon$ denotes the proportion of fertility reduction, the critical proportion of reduction, denoted by $\epsilon^{*}$, to lead to a demographic stationary state is determined by the equation $\left(1-\epsilon^{*}\right) R_{0}=1$, i.e.,

$$
\epsilon^{*}=1-\frac{1}{R_{0}},
$$

which is the demographic analogue of the famous control relation (the critical proportion of immunisation) in mathematical epidemiology. On the other hand, if $R_{0}<1$, the critical proportion of fertility increase to attain the replacement level is given by $1 / R_{0}-1$.

16 A mathematically rigorous treatment of the multistate strong ergodicity theorem can be found in Inaba $(1987,1988 \mathrm{a})$. An extension to the non-Markovian assumption is given in Inaba (1992). 
However, for the multiregional model, it is not easy to see the effect of changes in entries of the net reproduction matrix $K$ on $R_{0}$, and not only fertility but also the force of migration becomes a control parameter affecting the entries of $K$. Since the effects of changes in pattern and intensity of migration on $K$ are complex, in the following we mainly consider effects of fertility change under given multiregional survival rates.

Again let us consider the urban-rural (two-regional) net reproduction matrix as

$$
K=\left(\begin{array}{ll}
k_{11} & k_{12} \\
k_{21} & k_{22}
\end{array}\right),
$$

where we assume that $R_{0}=r(K)>1$. If all entries are uniformly reduced as much as $\epsilon$, it is clear that the multiregional net reproduction rate becomes $(1-\epsilon) r(K)$. In this uniform reduction case, the critical proportion of reduction $\epsilon^{*}$ with which a multiregional zero-growth population is maintained is again given by (4.1), while if the proportion of reduction is not uniform, it is not the case.

Suppose that fertility of rural area (Region 2) is reduced as much as $\epsilon \in(0,1)$, hence the controlled net reproduction matrix, denoted by $K(\epsilon)$, becomes

$$
K(\epsilon)=\left(\begin{array}{cc}
k_{11} & k_{12} \\
(1-\epsilon) k_{21} & (1-\epsilon) k_{22}
\end{array}\right) .
$$

Alternatively, if individuals born in rural areas reduce their fertility as much as $\epsilon$, then the controlled net reproduction matrix becomes

$$
K=\left(\begin{array}{ll}
k_{11} & (1-\epsilon) k_{12} \\
k_{21} & (1-\epsilon) k_{22}
\end{array}\right) .
$$

In both cases, the net reproduction rate is calculated as

$$
\begin{aligned}
R_{0} & =r(K(\epsilon)) \\
& =\frac{1}{2}\left[k_{11}+(1-\epsilon) k_{22}+\sqrt{\left(k_{11}+(1-\epsilon) k_{22}\right)^{2}-4(1-\epsilon) \operatorname{det} K}\right],
\end{aligned}
$$

which is a complex function of the reduction rate $\epsilon$. Since the critical proportion of fertility reduction $\epsilon^{*}$ is determined by $r\left(K\left(\epsilon^{*}\right)\right)=1$, there is no simple relation between $\epsilon^{*}$ and $R_{0}$. If the dimension of the state space becomes larger, it is almost impossible to give an explicit relationship between $R_{0}$ and the critical proportion of fertility reduction.

In order to obtain a simple control relation even for non-uniform changes in entries of the net reproduction matrix, we adopt a new reproduction number called the type-reproduction number introduced by Heesterbeek and Roberts (2003). ${ }^{17}$

17 Inaba and Nishiura (2008) formulated a similar type of reproduction number called the statereproduction number based on the multistate stable population model. The state-reproduction number coincides with the type-reproduction number when all states are birth state, while it can be applied to more general multistate models whose individual states are not necessarily birth 
The type-reproduction number for a target region is defined as the total number of female offspring in the target region produced by a woman born in the target region during her whole reproductive life. Here it must be noted that the typereproduction number takes into account not only newborns born directly to women in the target region but also newborns in the target region produced by way of descendants of those women who were born in non-target regions.

To explain the above definition, let us return to the urban-rural (two-regional) model (4.2). If $k_{11}<1$, i.e. if the urban-born population cannot replace itself without the rural area, the type-reproduction number of the rural region (target region), denoted by $T_{2}$, is calculated as

$$
T_{2}=k_{22}+\frac{k_{21} k_{12}}{1-k_{11}} .
$$

The first part $k_{22}$ denotes the total number of female newborns in the rural region directly produced by a woman born in the rural region, the second part

$$
\frac{k_{21} k_{12}}{1-k_{11}}=k_{21}\left(1+k_{11}+k_{11}^{2}+\cdots\right) k_{12},
$$

denotes the number of rural offspring by way of urban-born individuals who are descendants of rural-born women with no intermediate rural-born descendants. In fact, a rural-born woman produces $k_{12}$ female newborns in the urban area, an urban-born woman leaves $1 /\left(1-k_{11}\right)=1+k_{11}+k_{11}^{2}+\cdots$ descendants born in the urban area and each urban-born woman produces $k_{21}$ offspring in the rural area. As shown in Mathematical Appendix, we can prove that $R_{0}>1$ if $T_{2}>1, R_{0}=1$ if $T_{2}=1$ and $R_{0}<1$ if $T_{2}<1$, i.e. the threshold condition for population growth is expressed by $T_{2}=1$.

It is easily seen from (4.3) that if rural fertility decreases as much as $\epsilon$, i.e. if $k_{22}$ and $k_{21}$ are replaced by $(1-\epsilon) k_{22}$ and $(1-\epsilon) k_{21}$, respectively, or the fertility of females born in the rural area decreases as much as $\epsilon$, i.e. if $k_{22}$ and $k_{12}$ are replaced by $(1-\epsilon) k_{22}$ and $(1-\epsilon) k_{12}$, respectively, then $T_{2}$ becomes $(1-\epsilon) T_{2}$, in both cases. Therefore the critical reduction rate is simply calculated as

$$
\epsilon^{*}=1-\frac{1}{T_{2}}
$$

That is, if rural fertility decreases as much as $\epsilon^{*}$, or if the native-dependent net reproduction rate of the rural area is reduced as much as $\epsilon^{*}, T_{2}$ and $R_{0}$ become unity and the population converges to a stationary state.

state. The birth state is a state where a newborn can be produced. In the multiregional model, we usually assume that all regions are birth state, so we do not need to distinguish the typereproduction number and the state-reproduction number, but this is not necessarily the case for other multistate models. 
Next let us consider a case where two control programs are acting independently. Suppose that the rural fertility is decreased as much as $\epsilon_{1}$, and the native-dependent net reproduction rate of the rural area is reduced as much as $\epsilon_{2}$. Then the net reproduction matrix is changed into

$$
K\left(\epsilon_{1}, \epsilon_{2}\right)=\left(\begin{array}{cc}
k_{11} & \left(1-\epsilon_{2}\right) k_{12} \\
\left(1-\epsilon_{1}\right) k_{21} & \left(1-\epsilon_{1}\right)\left(1-\epsilon_{2}\right) k_{22}
\end{array}\right) .
$$

Then the type-reproduction number of the rural area is $\left(1-\epsilon_{1}\right)\left(1-\epsilon_{2}\right) T_{2}$, the critical reduction level, denoted by $\left(\epsilon_{1}^{*}, \epsilon_{2}^{*}\right)$, is a set given by a nonlinear relation as:

$$
\left(1-\epsilon_{1}^{*}\right)\left(1-\epsilon_{2}^{*}\right)=\frac{1}{T_{2}} .
$$

In such a case, though we no longer have a simple linear control relation, the control set of parameters given by (4.4) is still much simpler than the control set defined by $R_{0}=r\left(K\left(\epsilon_{1}, \epsilon_{2}\right)\right)=1$.

As a numerical example, let us consider an urban-rural model given by Rogers (1995, p. 128). Suppose that the next generation matrix is given by

$$
K=\left(\begin{array}{ll}
k_{11} & k_{12} \\
k_{21} & k_{22}
\end{array}\right)=\left(\begin{array}{cc}
\frac{3}{4} & \frac{1}{2} \\
\frac{1}{4} & 1
\end{array}\right) .
$$

Then the net reproduction rate of this two-region system is above replacement level:

$$
R_{0}=r(K)=\frac{1}{2}\left[k_{11}+k_{22}+\sqrt{\left(k_{11}-k_{22}\right)^{2}+4 k_{12} k_{21}}\right]=\frac{5}{4}>1 .
$$

Since $k_{11}<1$, we can calculate the type-reproduction number for the rural area as:

$$
T_{2}=k_{22}+\frac{k_{12} k_{21}}{1-k_{11}}=\frac{3}{2} \text {. }
$$

Therefore we know that $\epsilon^{*}=1-1 / T_{2}=1 / 3$, hence the total population will be controlled to a stationary state if rural fertility, or the rural-born individuals' fertility, decreases by as much as 33 per cent. On the other hand, the total population will go to a stationary state if we decrease everyone's fertility by as much as $\left(1-1 / R_{0}\right) \times 100=20$ per cent. 


\section{Discussion}

The type-reproduction number of a target region is not finitely defined if the net reproduction rate of the multiregional system composed of non-target regions is not less than unity. However, for example, as is observed in many developed countries (such as EU countries and Japan), not only the national fertility is below replacement level but all regional, subnational or prefectural fertilities as well. In such a case, we can compute the type-reproduction number for each region, ${ }^{18}$ and its theory tells us that we can increase the national (or multinational) fertility rate to above replacement level by increasing the fertility of some target regions intensively, instead of increasing every regional (or cohort) fertilities uniformly. This multistate perspective reflecting demographic heterogeneity is crucially important for discussing multiregional population policy, because uniformly effective control is not realistic.

As is shown above, the type-reproduction number gives a useful index to formulate the critical fertility level of individuals at specific target states to which population control policy will be applied, provided that migration pattern and intensity are given. It is a future challenge to examine the effects of variation of migration on the net reproduction matrix and $R_{0}$. On the other hand, as we see in the Appendix, we can formulate the renewal equation for the target population to calculate other demographic indices, including the generation time, the intrinsic growth rate and the momentum of population growth, which are also useful to study the impact of heterogeneous individual behaviour on population dynamics as a whole. Historically speaking, as Heesterbeek (2002) pointed out, the concept of the net reproduction rate $R_{0}$ was already established at least in 1925 by Dublin and Lotka (Dublin and Lotka 1925; Lotka 1998) in demography, ${ }^{19}$ while it has taken more than 50 years for the concept to mature in epidemiology. Moreover it was not until recently that stable population theory became a popular tool in mathematical epidemiology (Wallinga and Lipsitch 2007) and I believe that there are still many ideas and concepts in demography that should be learned by epidemiologists. However, the progress of mathematical epidemiology during the last decade is remarkable, hence in some aspects $R_{0}$ is more fully developed in epidemiology than in demography. The type-reproduction number (or the statereproduction number) is a typical result of those epidemiological developments in

18 In the $N$-regional system, if the target region is one, its type-reproduction number $T_{1}$ is given by the Roberts-Heesterbeek formula (Roberts and Heesterbeek 2003) as

$$
T_{1}=r\left(P K(I-(I-P) K)^{-1}\right)=e_{1}^{T} P K(I-(I-P) K)^{-1} e_{1},
$$

where $P$ is a projection matrix to the first state (that is, the (1,1)-th entry is unity and all other entries are zero) and $e_{1}$ is a unit vector whose entries other than the first entry are all zero, provided that $r((I-P) K)<1$, that is, the net reproduction rate of multiregional system excluding the first state is below replacement level.

19 In fact, the roots of $R_{0}$ could be traced back to much earlier time (almost 18th century), and epidemic $R_{0}$ has its roots in 19th century (Nishiura et al. 2006; Nishiura and Inaba 2007). 
basic reproduction number theory. Now it would be the turn of demography to import epidemiological ideas. Just as in mathematical epidemiology, the net reproduction rate $R_{0}$ must become a key concept to reactivate multistate mathematical demography, and then it would prove to be a useful tool for investigating multinational population dynamics with migration.

\section{References}

Billari, F. C., P. Manfredi, and A. Valentini. 2000. "Macro-demographic effects of the transition to adulthood: Multistate stable population theory and an application to Italy." Mathematical Population Studies 9(1): 33-63.

Coleman, D. 2006. "Immigration and ethnic change in low-fertility countries: A third demographic transition." Population and Development Review 32(3): 401-446.

Diekmann, O., J. A. P. Heesterbeek, and J. A. J. Metz. 1990. "On the definition and the computation of the basic reproduction ratio $R_{0}$ in models for infectious diseases in heterogeneous populations." Journal of Mathematical Biology 28: 365-382.

Diekmann, O. and J. A. P. Heesterbeek. 2000. Mathematical Epidemiology of Infectious Diseases: Model Building, Analysis and Interpretation. Chichester, John Wiley and Sons.

Dietz, K. 1993. "The estimation of the basic reproduction number for infectious diseases." Statistical Methods in Medical Research 2: 23-41.

Dublin, L. I. and A. J. Lotka. 1925. "On the true rate of natural increase." Journal of the American Statistical Association New Series 150(20): 305-339.

Heesterbeek, J. A. P. 2002. "A brief history of $R_{0}$ and a recipe for its calculation." Acta Biotheoretica 50: 189-204.

Heesterbeek, J. A. P. and M. G. Roberts. 2007. "The type-reproduction number $T$ in models for infectious disease control." Mathematical Biosciences 206: 3-10.

Inaba, H. 1986. "On the discrete model of multiregional demographic growth." Journal of Population Problems 179: 1-15. [in Japanese with English summary]

Inaba, H. 1987. "Mathematical foundation of multidimensional stable population theory I: Classical theory." Journal of Population Problems 184: 52-77. [in Japanese with English summary]

Inaba, H. 1988a. "A semigroup approach to the strong ergodic theorem of the multistate stable population process." Mathematical Population Studies 1(1): 49-77.

Inaba, H. 1988b. "Asymptotic properties of the inhomogeneous Lotka-Von Foerster system." Mathematical Population Studies 1(3): 247-264.

Inaba, H. 1992. "Duration-dependent multistate population dynamics." Working Paper Series, No. 9, Institute of Population Problems, Tokyo.

Inaba, H. 1995. "Human population reproduction via first marriage." Mathematical Population Studies 5(2): 123-144.

Inaba, H. and H. Nishiura. 2008. "The state-reproduction number for a multistate class age structured epidemic system and its application to the asymptomatic transmission model." Mathematical Biosciences 216: 77-89.

Keyfitz, N. and H. Caswell. 2005. Applied Mathematical Demography. 3rd Edition, New York, Springer. 
Land, K. C. and A. Rogers (eds.) 1982. Multidimensional Mathematical Demography. New York, Academic Press.

Le Bras, H. 1971. "Equilibre et croissance de populations soumises à des migrations." Theoretical Population Biology 2: 100-121.

Lotka, A. J. 1998. Analytical Theory of Biological Populations. Translated and with an introduction by D. P. Smith and H. Rossert. New York and London, Plenum Press. [translation from original edition Théorie analytique des associations biologiques. Deuxième partie: Analyse démographique avec application particulière à l'espèce humaine. (Actualités Scientifiques et Industrielles, No. 780), Paris, Hermann et Cie, 1939.]

Metz, J. A. J. and O. Diekmann. 1986. The Dynamics of Physiologically Structured Populations. Lecture Notes in Biomathematics 68, Berlin, Springer.

Minc, H. 1988. Nonnegative Matrices. New York, John Wiley and Sons.

Nishiura, H., K. Dietz, and M. Eichner. 2006. "The earliest notes on the reproduction number in relation to herd immunity: Theophil Lotz and smallpox vaccination." Journal of Theoretical Biology 241: 964-967.

Nishiura, H. and H. Inaba. 2007. "Discussion: Emergence of the concept of the basic reproduction number from mathematical demography." Journal of Theoretical Biology 244: 357-364.

Preston, S. H., P. Heuveline, and M. Guillot. 2001, Demography: Measuring and Modeling Population Processes. Oxford, Blackwell.

Roberts, M. G. and J. A. P. Heesterbeek. 2003. "A new method for estimating the effort required to control an infectious disease." Proceedings of the Royal Society of London B 270: 1359-1364.

Rogers, A. 1966. "The multiregional matrix growth operator and the stable interregional age structure." Demography 3: 537-544.

Rogers, A. 1968. Matrix Analysis of Interregional Population Growth and Distributions. Berkeley, University of California Press.

Rogers, A. 1975. Introduction to Multiregional Mathematical Demography. New York, John Wiley.

Rogers, A. and F. Willekens. 1978. "The spatial reproductive value and the regional momentum of zero population growth." Environment and Planning A 10: 503-518.

Rogers, A. and F. J. Willekens (eds.) 1986. Migration and Settlement: A Multiregional Comparative Study. Dordrecht, D. Reidel.

Rogers, A. 1995. Multiregional Demography: Principles, Methods and Extensions. New York, John Wiley.

Schoen, R. 1988. Modeling Multigroup Populations. New York and London, Plenum Press.

Wallinga, J. and M. Lipsitch. 2007. "How generation intervals shape the relationship between growth rates and reproductive numbers." Proceedings of the Royal Society B 274: 599-604.

Willekens, F. 1977. "The spatial reproductive value: theory and applications." RM-77-9, Laxenburg, IIASA. 


\section{Mathematical appendix}

Here we briefly sketch the dynamical system formulation for the typereproduction number and its computation method. Although the type-reproduction number theory is formulated for any number of target states based on the general $\mathrm{N}$-dimensional renewal system (Inaba and Nishiura 2008), for illustrative purposes, we here consider a two-regional stable population system:

$$
B_{1}(t)=G_{1}(t)+\int_{0}^{t} \psi_{11}(a) B_{1}(t-a) d a+\int_{0}^{t} \psi_{12}(a) B_{2}(t-a) d a,
$$

$$
B_{2}(t)=G_{2}(t)+\int_{0}^{t} \psi_{21}(a) B_{1}(t-a) d a+\int_{0}^{t} \psi_{22}(a) B_{2}(t-a) d a,
$$

where $\psi_{i j}(a)$ is the $(i, j)$-th entry of the 2-regional net maternity function matrix $\Psi(a)$ and

$$
k_{i j}=\int_{0}^{\infty} \psi_{i j}(a) d a,
$$

gives the $(i, j)$-th entry of the 2-regional net reproduction matrix $K$.

If we use a symbol for convolution integral as

$$
(f * g)(t)=\int_{0}^{t} f(a) g(t-a) d a,
$$

then (6.1) is simply expressed as follows:

$$
\begin{aligned}
& B_{1}=G_{1}+\psi_{11} * B_{1}+\psi_{12} * B_{2}, \\
& B_{2}=G_{2}+\psi_{21} * B_{1}+\psi_{22} * B_{2} .
\end{aligned}
$$

In the following, we assume that $k_{22}<1$, i.e. the net reproduction rate is below replacement level if a woman spends her entire reproductive life stage in the second region. Under this basic assumption, first let us solve the renewal equation of newborns in the second region. If we see $B_{1}(t)$ as a given function, we can formally solve the renewal equation for $B_{2}$ as follows:

$$
\begin{aligned}
B_{2} & =G_{2}+\psi_{21} * B_{1}+\phi_{22} *\left(G_{2}+\psi_{21} * B_{1}\right) \\
& =G_{2}+\phi_{22} * G_{2}+\left(\psi_{21}+\phi_{22} * \psi_{21}\right) * B_{1}
\end{aligned}
$$

where $\phi_{22}$ is the resolvent kernel corresponding to $\psi_{22}$ defined by the solution of the resolvent equation:

$$
\phi_{22}=\psi_{22}+\phi_{22} * \psi_{22} \text {. }
$$


If we insert the expression (6.3) into the renewal equation for $B_{1}$ in (6.2), we obtain a new renewal equation for $B_{1}$ :

(6.5) $B_{1}=G_{1}+\psi_{11} * B_{1}+\psi_{12} *\left[G_{2}+\phi_{22} * G_{2}+\left(\psi_{21}+\phi_{22} * \psi_{21}\right) * B_{1}\right]$

$$
=G_{1}+\psi_{12} * G_{2}+\psi_{12} *\left(\phi_{22} * G_{2}\right)+\left[\psi_{11}+\psi_{12} *\left(\psi_{21}+\phi_{22} * \psi_{21}\right)\right] * B_{1}
$$

Let us define the integral kernel and the initial data for the reduced system as

$$
\begin{aligned}
& \pi_{1}:=\psi_{11}+\psi_{12} *\left(\psi_{21}+\phi_{22} * \psi_{21}\right), \\
& H_{1}:=G_{1}+\psi_{12} * G_{2}+\psi_{12} *\left(\phi_{22} * G_{2}\right) .
\end{aligned}
$$

From (6.5) and (6.6), we arrive at a single renewal equation for $B_{1}$ :

$$
B_{1}(t)=H_{1}(t)+\int_{0}^{t} \pi_{1}(a) B_{1}(t-a) d a,
$$

where the integral kernel $\pi_{1}$ describes not only the number of female children born in the first region to a woman born in the first region, but also the number of progeny born in the first region to females born in the second region whose family line started from individuals born in the first region without intermediate descendants born in the first region. Once we can determine the birth trajectory of the first-region population by (6.7), the birth trajectory of the second-region population is given by (6.3).

Based on the renewal process described by (6.7), we define the typereproduction number for the first-region population, denoted by $T_{1}$, as

$$
T_{1}=\int_{0}^{\infty} \pi_{1}(a) d a
$$

From (6.6), we can calculate $T_{1}$ as

$$
T_{1}=k_{11}+k_{12} k_{21}\left(1+\int_{0}^{\infty} \phi_{22}(a) d a\right) .
$$

By integrating both sides of the resolvent Equation (6.4), we have

$$
\int_{0}^{\infty} \phi_{22}(a) d a=k_{22}\left(1+\int_{0}^{\infty} \phi_{22}(a) d a\right)
$$

where the integrability of the resolvent kernel $\phi_{22}$ is guaranteed by our assumption that $k_{22}<1$ (see Inaba and Nishiura 2008, Paley-Wiener Theorem). Then we have

$$
\int_{0}^{\infty} \phi_{22}(a) d a=\frac{k_{22}}{1-k_{22}}
$$


Inserting the above expression into (6.8), we obtain

$$
T_{1}=k_{11}+\frac{k_{12} k_{21}}{1-k_{22}} .
$$

Then we can easily confirm that if $T_{1}>1$, then $R_{0}>1$, if $T_{1}=1$, then $R_{0}=1$ and if $T_{1}<1$, then $R_{0}<1$. In fact, let $f(\lambda)=\operatorname{det}(\lambda I-K)$. Then $R_{0}$ is the largest positive root of $f(\lambda)=0$ and it follows that

$$
f(1)=\left(1-k_{22}\right)\left(1-T_{1}\right), \quad f^{\prime}(1)=2-\left(k_{11}+k_{22}\right) .
$$

If $T_{1}>1$, then $f(1)<0$ and $f(\lambda)=0$ has a positive root larger than unity, we conclude that $R_{0}>1$. If $T_{1}=1$, then $f(1)=0$ and $f^{\prime}(1)>0$ because $k_{22}<1$ and $k_{11}$ $<T_{1}=1$. Then the largest positive root of $f(\lambda)=0$ is unity, i.e. $R_{0}=1$.

Finally if $T_{1}<1$, then $f(1)>0$ and $f^{\prime}(1)>0$, which implies that $R_{0}<1$. The reader may refer to Roberts and Heesterbeek (2003) and Inaba and Nishiura (2008) for a general computation method of the type-reproduction (or statereproduction) number in the $\mathrm{N}$-dimensional case. 